This is the accepted manuscript made available via CHORUS, the article has been published as:

Electric field modulation of the tetragonal domain orientation revealed in the magnetic ground state of quantum paraelectric EuTiO_\{3\}

A. P. Petrović, Y. Kato, S. S. Sunku, T. Ito, P. Sengupta, L. Spalek̄, M. Shimuta, T. Katsufuji, C. D. Batista, S. S. Saxena, and C. Panagopoulos Phys. Rev. B 87, 064103 - Published 8 February 2013

DOI: 10.1103/PhysRevB.87.064103 


\title{
Electric Field Modulation of the Tetragonal Domain Orientation Revealed in the Magnetic Ground State of Quantum Paraelectric $\mathrm{EuTiO}_{3}$
}

\author{
A.P. Petrović, ${ }^{1}$ Y. Kato, ${ }^{2}$ S.S. Sunku, ${ }^{1}$ T. Ito,${ }^{3}$ P. Sengupta, ${ }^{1}$ L. Spalek, ${ }^{4,5}$ M. \\ Shimuta, ${ }^{6}$ T. Katsufuji, ${ }^{6}$ C.D. Batista, ${ }^{2}$ S. Saxena, ${ }^{4}$ and C. Panagopoulos ${ }^{1,4,5}$ \\ ${ }^{1}$ Division of Physics and Applied Physics, Nanyang Technological University, 637371 Singapore \\ ${ }^{2}$ Theoretical Division, CNLS and T-4, Los Alamos National Laboratory, Los Alamos, New Mexico 87545, USA \\ ${ }^{3}$ National Institute of Advanced Industrial Science and Technology (AIST), Ibaraki 305-8562, Japan \\ ${ }^{4}$ Cavendish Laboratory, University of Cambridge, Cambridge CB3 OHE, United Kingdom \\ ${ }^{5}$ Department of Physics, University of Crete and FORTH, 71003 Heraklion, Greece \\ ${ }^{6}$ Department of Physics, Waseda University, Tokyo 169-8555, Japan
}

(Dated: January 7, 2013)

\begin{abstract}
We present a study of the thermodynamic and magnetic properties of single-crystal $\mathrm{EuTiO}_{3}$. Signatures of metastability are visible in the heat capacity below the cubic-tetragonal phase transition at $283 \mathrm{~K}$, supporting the evidence for a mismatch between long and short range structural order from previous X-ray diffraction studies. Employing the anisotropic magnetization as an indirect structural probe, we confirm the emergence of multiple orthogonal domains at low temperature. Torque magnetometry is capable of revealing the nature and temperature-dependence of the magnetic anisotropy in spite of the domain misalignment; we hence deduce that tetragonal $\mathrm{EuTiO}_{3}$ enters an easy-axis antiferromagnetic phase at $5.6 \mathrm{~K}$, with a first-order phase transition to an easyplane ground state below $3 \mathrm{~K}$. Our experimentally-determined magnetic phase diagram is accurately reproduced by a 3D anisotropic Heisenberg spin model. Furthermore, we demonstrate that electric field cooling acts to suppress this orientational disorder by realigning the domains, due to the strong coupling between electric fields and lattice dipoles characteristic of paraelectric materials.
\end{abstract}

PACS numbers: 75.85.+t, 75.30.Gw, 64.60.Cn, 75.10.Dg

\section{INTRODUCTION}

Perovskite titanates $A \mathrm{TiO}_{3}$ are among the brightest stars in the rapidly-developing field of oxide electronics. Their dielectric and transport properties are easily modulated by epitaxial strain, field-effect or chemical doping ${ }^{1-4}$, hence facilitating their integration with conventional charge-based electronics. However, the past decade has also seen an intensive search for a multiferroic perovskite with strong magnetoelectric coupling, which would enjoy numerous spintronic applications.

The spotlight soon fell on $\mathrm{EuTiO}_{3}$, whose large magnetic moment $\left(S=7 / 2\right.$ per unit cell from the $4 \mathrm{f}^{7} \mathrm{Eu}^{2+}$ ion) and quantum paraelectricity suggest that it is a magnetic analogue of $\mathrm{SrTiO}_{3}$, the "workhorse" oxide for electronic devices. Although $\mathrm{EuTiO}_{3}$ has been known as a $G$-type antiferromagnet $(\mathrm{AF})$ below $\mathrm{T}_{N} \sim 5.5 \mathrm{~K}$ since the $1960 \mathrm{~s}^{5}$, its magnetoelectric properties were only revealed in 2001 by a $7 \%$ drop in the dielectric constant at $\mathrm{T}_{N}{ }^{6}$.

Early efforts to model $\mathrm{EuTiO}_{3}$ shared one critical feature: an assumption of cubic crystal symmetry throughout the phase diagram ${ }^{7-9}$. The overwhelming majority of cubic perovskites undergo phase transitions to tetragonal or orthorhombic structures via stretching or rotation of their oxygen octahedra; however the resultant change in lattice parameters is often extremely subtle and the true structural ground state is difficult to determine using either conventional X-ray diffraction (XRD) or thermodynamic techniques. $\mathrm{SrTiO}_{3}$ provides an excellent example of such challenges: despite being one of the most popular and widely-used substrates for complex oxide film growth over the past 25 years, its cubictetragonal transition at $105 \mathrm{~K}$ was only detected thermodynamically in $1998^{10}$. Very recently, evidence has been uncovered for a cubic-tetragonal transition at $283 \mathrm{~K}$ in powdered samples of $\mathrm{EuTiO}_{3}{ }^{11,12}$. This revelation immediately obliges us to revisit our understanding of the low-temperature properties of $\mathrm{EuTiO}_{3}$, since tetragonal symmetry pushes the material closer to a ferromagnetic/ferroelectric $(\mathrm{FM} / \mathrm{FE})$ phase boundary ${ }^{7}$, as well as permitting the breakage of spatial inversion symmetry and introducing an anisotropy into any magneticallyordered phase. Preliminary work suggests more complex low-temperature behaviour compared with $\mathrm{SrTiO}_{3}$, with XRD analysis even suggesting that tetragonal $\mathrm{EuTiO}_{3}$ may be intrinsically disordered ${ }^{13}$. Theoretical studies show that $\mathrm{EuTiO}_{3}$ is elastically harder than $\mathrm{SrTiO}_{3}$, which is also conducive to disorder ${ }^{14}$.

The presence of disorder in a magnetoelectric such as $\mathrm{EuTiO}_{3}$ would open the door to local symmetry-breaking and electric dipole formation via the DzyaloshinskiiMoriya (DM) interaction. This could generate nanoscale ferroelectric domains with a strong magnetoelectric coupling. More generally, nanoscale heterogeneity limits applications for many complex oxides ${ }^{15}$, since functional electronic materials must display phase purity over lengthscales greater or equal to their intended device dimensions. As well as detecting the presence of structural disorder and determining its effects on the useful properties of such materials, it is therefore an urgent priority to identify methods for controlling or suppressing 
such disorder. In this report, we utilize the low temperature dc magnetization of antiferromagnetic $\mathrm{EuTiO}_{3}$ as a structural probe, hence showing that monocrystalline cubic $\mathrm{EuTiO}_{3}$ undergoes a transition to a tetragonal phase with multiple domains which may be distinguished by their orthogonal tetragonal $c$ axes. This structure can be considered to be "orientationally disordered". Torque magnetometry is impervious to the presence of domains, enabling us to extract the magnetic anisotropy and map out the magnetic phase diagram of $\mathrm{EuTiO}_{3}$, which corresponds accurately to the phase diagram calculated using a classical Heisenberg spin model. Upon recooling the same crystals under an electric field, a realignment of the domains occurs which reduces the orientational disorder. This implies that the electric field directly influences the low temperature crystal structure by suppressing twinning and other lattice imperfections linked to the presence of domains. We will briefly discuss the implications and potential for this method of homogeneity control.

\section{METHODS AND TECHNIQUES}

Our experiments were performed on $\mathrm{EuTiO}_{3}$ single crystals, grown in a floating-zone furnace ${ }^{16}$. Highsymmetry axes were determined by XRD in the cubic phase at $300 \mathrm{~K}$ and the crystals cut into cuboids of mass $\sim 4 \mathrm{mg}$, with faces parallel to (100), (010) and (001). The cutting/alignment error is $\sim \pm 5^{\circ}$, a figure supported by our torque data. A Laue camera image of one of the crystals is shown in Fig. 1: we stress that at $300 \mathrm{~K}$, our samples are perfectly monocrystalline with no evidence for twinning.

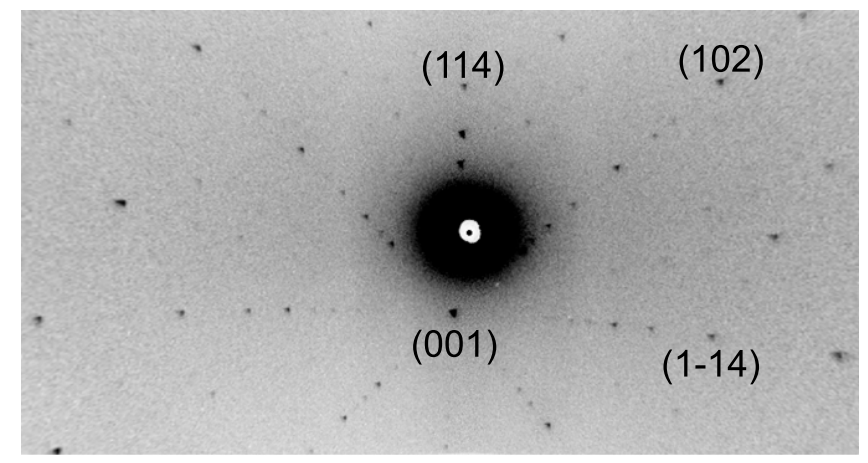

FIG. 1: Laue photograph of a typical $\mathrm{EuTiO}_{3}$ crystal at $300 \mathrm{~K}$, acquired prior to cutting and alignment. The indices of several diffraction planes are indicated; no splitting of the diffraction spots is discernible.

The heat capacity and magnetic torque were measured using a Quantum Design PPMS, while the dc magnetization was acquired using a MPMS XL SQUID magnetometer from the same manufacturer. Geometric demagnetization corrections ${ }^{17}$ have been applied to all our data, except the specific heat and torque. Given the large magnetic moment in $\mathrm{EuTiO}_{3}$, it is essential to include the demagnetization factor in order to perform any meaningful quantitative comparison between experiment and theory. Our results have been verified using four separate crystals, all of which display similar behaviour.

\section{RESULTS AND DISCUSSION}

\section{A. Heat capacity}
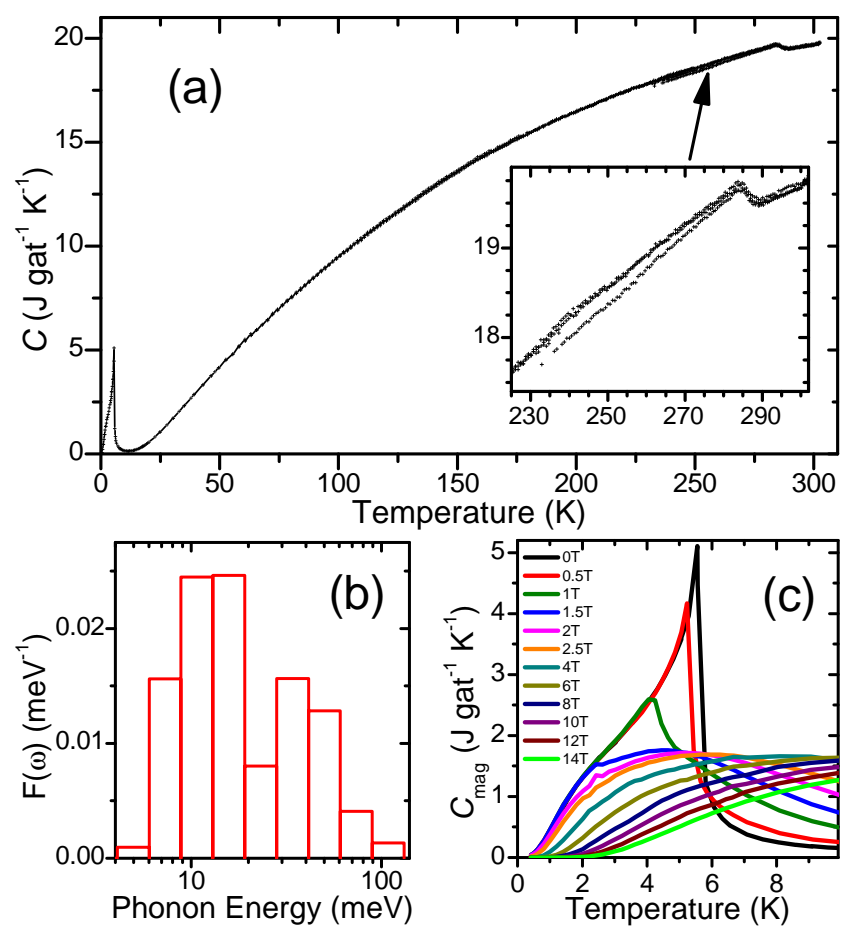

FIG. 2: (a) Zero-field heat capacity $C(T)$ in $\mathrm{EuTiO}_{3}$. Inset: zoom on the structural transition at $283 \mathrm{~K}$, showing two divergent traces generated by consecutive thermal relaxations. (b) PDOS extracted from the specific heat (see ref. ${ }^{18}$ for details). (c) Low temperature magnon heat capacity $C_{\text {mag }}$, illustrating the field-suppression of AF order and spin gap formation. The absence of the spin-flop and spin realignment transitions from $C(T, H)$ is unsurprising, since the entropy change is extremely small and short-pulse relaxation calorimetry is notoriously insensitive to first-order transitions ${ }^{19}$. Data are qualitatively similar regardless of the field orientation, hence we only display $H / /[010]$ for clarity.

The structural transition in $\mathrm{EuTiO}_{3}$ is visible as a kink in the heat capacity $C(T)$ at $T_{c}=283 \mathrm{~K}$ (Fig. 2a). As we sweep the temperature downwards through a series of setpoints below $T_{c}$, repeated thermal excitation/relaxation measurement cycles yield two divergent traces in $C(T)$, the lower of which is generated by the first cycle at any given temperature and vanishes below $\sim 240 \mathrm{~K}$. This trend is reproducible for all our crystals, but absent from reference materials tested on the same calorimeter and hence not an experimental artifact. Such behaviour 
is suggestive of phase metastability in this temperature range.

Interestingly, XRD on powder samples indicates a lower $T_{c}$ of $\sim 235 \mathrm{~K}^{13}$, coinciding with the disappearance of our lower trace and providing further evidence for a non-equilibrium, evolving crystal structure below $283 \mathrm{~K}$. We also extract the temperature-integrated phonon density of states (PDOS) from $C(T)$ (Fig. 2b) and note the broad spectral weight distribution below $20 \mathrm{meV}$ : this corresponds to the TO1 phonon softening from $120 \mathrm{~cm}^{-1}$ to $80 \mathrm{~cm}^{-120}$.

Turning our attention to lower temperatures, we obtain the magnon heat capacity $C_{\mathrm{mag}}$ by subtracting a $\beta_{1} T^{3}+\beta_{2} T^{5}$ phonon background from $C(T)$ (Fig. 2c). A large jump at $T_{N}=5.6 \mathrm{~K}$ indicates a second-order $\mathrm{AF}$ transition, similar to that seen in existing powder ${ }^{11}$ and nanoparticle ${ }^{21}$ data. Integrating $C_{\mathrm{mag}} / T$ from zero to $5.6 \mathrm{~K}$ yields a total entropy of $69.4 \pm 1 \mathrm{mJgat}^{-1} \mathrm{~K}^{-1}$ which agrees with the theoretical value $N k_{B} \ln (2 S+1)$ $=69.8 \mathrm{mJgat}^{-1} \mathrm{~K}^{-1}$ and discourages the possibility of any further structural transition at $\mathrm{T} \leq 5.6 \mathrm{~K}$. Magnetic fields suppress $T_{N}$, inducing a $T=0$ transition (at $H=H_{\text {sat }} \simeq 1 \mathrm{~T}$ ) to saturated paramagnetism, with an energy gap for spin excitations growing linearly in $H-H_{\text {sat }}$. This confirms the phase purity of our samples, since any oxygen deficiency should lead to a reduction in $T_{N}$ and eventually drive a zero-field crossover from $\mathrm{AF}$ to FM order ${ }^{22}$.

\section{B. dc magnetization}

The tetragonal crystal structure in $\mathrm{EuTiO}_{3}$ must generate an anisotropic magnetic ground state and the first evidence for this emerges in the dc magnetic susceptibility $\chi=M / H$ (Fig. 3a), where three energy scales may be identified. The peak in $\chi(T)$ at $T_{N}$ is robust at low fields; however a "shoulder" forms below $T_{N}$, broadening and moving to lower temperature as $H$ increases. A third anomaly is visible below $3 \mathrm{~K}$, vanishing above $H \sim 0.1 \mathrm{~T}$. The magnetization saturates at a similar field $H_{\text {sat }} \sim 1 \mathrm{~T}$ along all three axes (Fig. 3b), with a moment $m_{\text {sat }}=7.08 \pm 0.2 \mu_{B} /$ unit cell. The slight anisotropy in $m_{\text {sat }}$ arises from an anisotropic $g$-tensor in this tetragonal system. At low fields, small anomalies in $M(H)$ are visible and $\chi(H)$ falls as $H$ tends to zero (Fig. 3c). The temperature and field-dependence of these non-linearities in $M(H)$ correlate with the "shoulder" in $\chi(T)$, leading us to identify them as spin-flop transitions.

However, a single-crystal AF with tetragonal symmetry should only exhibit a spin-flop when $\boldsymbol{H}$ is parallel to the $a b$ plane (for easy-plane spin orientation) or when $\boldsymbol{H}$ is parallel to the $c$ axis (for easy-axis alignment). In contrast, our crystals consistently display spin-flops at similar fields $\left(H_{s f}=0.22 \pm 0.04 \mathrm{~T}\right.$ at $\left.2 \mathrm{~K}\right)$ along all three axes. The spin configuration of a material must directly reflect its crystal symmetry and hence the only explanation for our results is the presence of multiple structural domains at low temperature in $\mathrm{EuTiO}_{3}$. Experimentally, we are measuring a superposition of $M(H)$ curves from orthogonal tetragonal domains. Similar values for $H_{\text {sat }}$ regardless of the direction of the applied magnetic field imply either a rather small anisotropy or an approximately equal distribution of domain orientations (or a combination of both factors). Unfortunately, our experiments do not permit us to estimate the lengthscale of these domains; however, we note that spontaneous twinning is observed in elastically soft $\mathrm{SrTiO}_{3}$ with domain size below $1 \mu \mathrm{m}^{23}$. The presence of a spin-flop in a $200 \mathrm{~nm} \mathrm{EuTiO}$ film also suggests that true nanoscale domains exist in this material ${ }^{24}$.
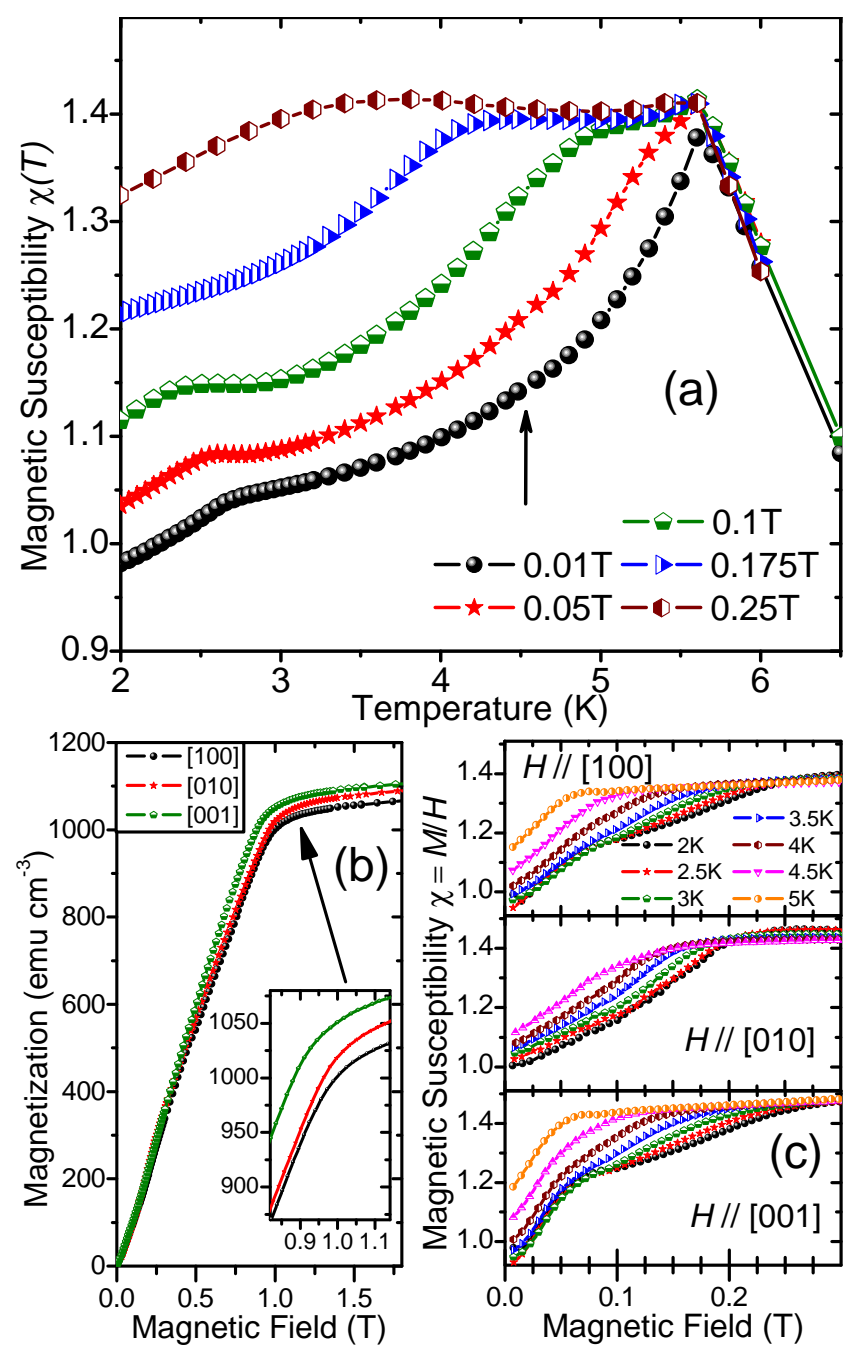

FIG. 3: (a) dc magnetic susceptibility $\chi(T)$ for a range of applied fields, revealing the transition to $a b$-plane order below $\sim 3 \mathrm{~K}$, a "shoulder" due to the spin-flop and a peak at $\mathrm{T}_{N}$. A faint anomaly is also visible in low fields around $4.5 \mathrm{~K}$ (black arrow). Data are qualitatively similar regardless of the field orientation, hence we only display $H / /$ [010] for clarity. (b) dc magnetization $M(H)$ along the crystal axes. (c) Spin-flop transitions in $\chi(H)$ for fields parallel to each axis. In the absence of a spin-flop, $\frac{\delta \chi}{\delta H}$ should remain constant down to $H=0$ (as illustrated by a dashed line for $\boldsymbol{H} / /[010]$ ). 


\section{Torque magnetometry}

Although we are able to confidently identify the AF transition and spin-flops using the dc magnetization, the presence of orthogonal domains prevents us from determining the nature of the third anomaly below $3 \mathrm{~K}$ at low fields. This anomaly is linked to the magnetic anisotropy and is hence key to our understanding of the low temperature spin structure and magnetic ground state. Since europium compounds exhibit significant neutron absorption, they are poorly-suited to neutron diffraction experiments (the standard method for resolving microscopic spin configurations). Torque magnetometry is therefore our best alternative to elucidate the spin structure and has previously been successfully applied to several antiferromagnetic materials in the literature ${ }^{25,26}$. The magnetic anisotropy may be determined by rotating a crystal within a constant magnetic field and tracking the temperature-dependence of both the amplitude and periodicity of the generated torque signal, as outlined in Fig. 4. An easy-axis two-sublattice AF (Fig. 4a) will generate an out-of-plane torque signal $\tau(\phi) \propto \sin 2 \phi$ when a field $\boldsymbol{H}$ rotates in a plane containing the easy axis; however $\tau=0$ if $\boldsymbol{H}$ lies in the plane perpendicular to the easy axis. Conversely, an easy-plane AF (Fig. 4b) will display $\tau(\theta) \propto \sin 4 \theta$ when $\boldsymbol{H}$ is rotated in the easy plane and $\tau(\phi) \propto \sin 2 \phi$ when $\boldsymbol{H}$ is rotated through any plane containing the hard axis ( $\phi$ and $\theta$ are the polar and azimuthal angles respectively). Anisotropic paramagnetism above $T_{N}$ will also generate a $\tau(\phi) \propto \sin 2 \phi$ signal.

Our magnetization data imply that $\mathrm{EuTiO}_{3}$ crystals contain AF domains with the easy-axis pointing along all three crystallographic axes. Therefore, the torque should behave as $\tau(\alpha)=A \sin 2 \alpha+B \sin 4 \alpha$, where $\alpha$ is the field angle in a plane defined by any pair of crystal axes. (The demagnetization factor also contributes to $\tau(\alpha)$ with $180^{\circ}$ periodicity, but it is temperature invariant and does not affect our conclusions.) A series of $\tau(\alpha)$ curves for $2 \mathrm{~K} \leq T \leq 15 \mathrm{~K}$ is shown in Fig. 4c, with $H=0.05$ T rotating in the (001) plane: the transition from $\sin 2 \alpha$ behaviour above $T_{N}$ to $\sin 4 \alpha$ at low temperature is clearly visible. Fitting our $\tau(\alpha)$ curves to obtain the $\sin 2 \alpha$ and $\sin 4 \alpha$ weight parameters $A$ and $B$, we note that $B$ only appears below $T_{N}$, rising rapidly below $3 \mathrm{~K}$ with a corresponding drop in $A$. The only mechanism which can generate this behaviour is a change from easy-axis to easy-plane symmetry: we deduce that a first-order phase transition from $c$-axis to $a b$-plane $\mathrm{AF}$ ordering occurs at $T_{a b}$ between $2.75 \mathrm{~K}$ and $3 \mathrm{~K}$. Upon increasing $H$ (Fig. 4e), $\tau(\alpha)$ changes shape from sinusoidal to "sawtooth", indicating a spin-flop" 26 . At $H=0.25 \mathrm{~T}$, the approximate $\sin 2 \alpha$ dependence is characteristic of a spin-flopped AF.
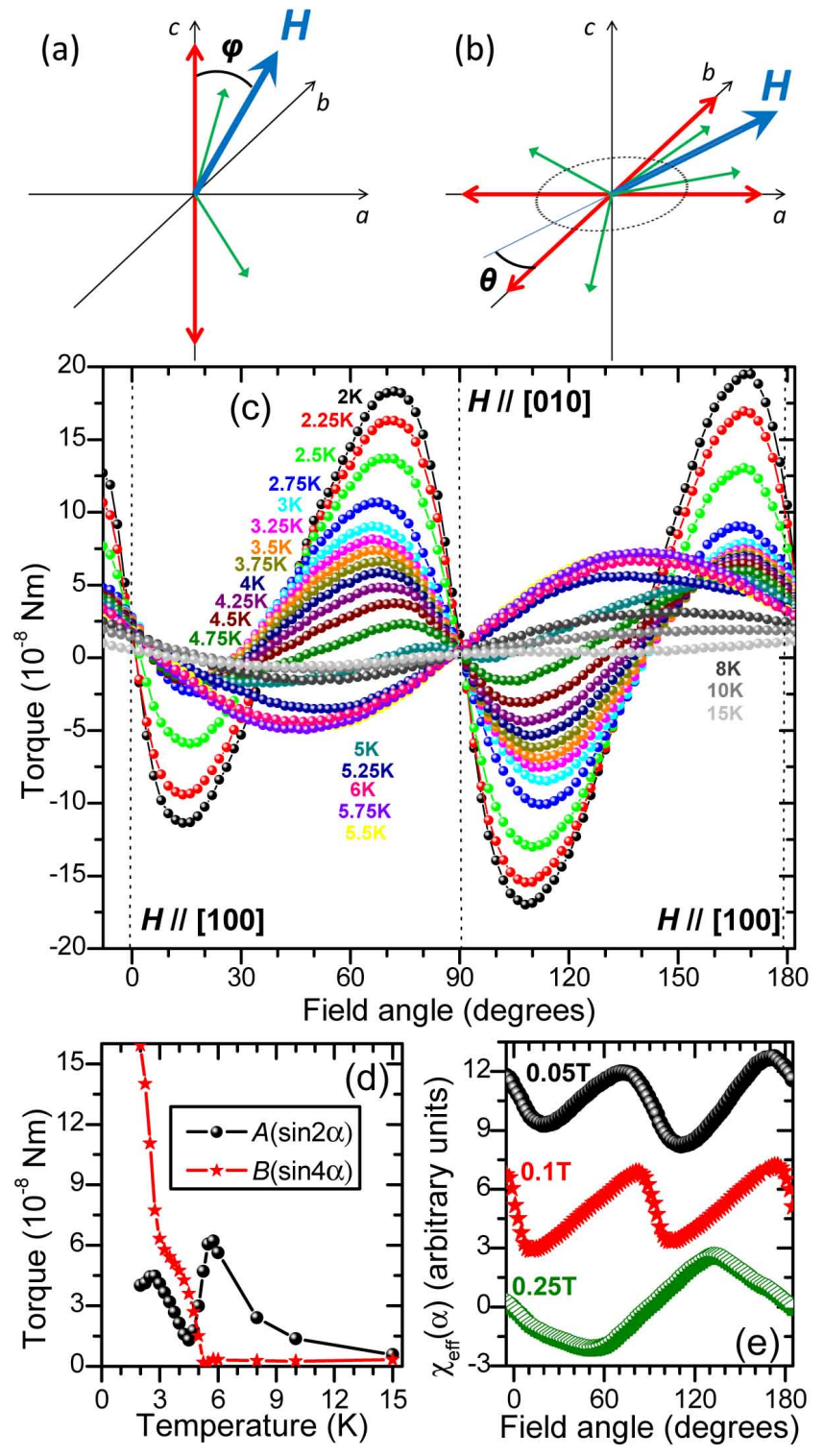

FIG. 4: (a,b) Torque experimental schematic, with easy axes shown in red, AF sublattice moments in green and field $\boldsymbol{H}$ in blue. (c) Temperature dependence of $\tau(\alpha) / /$ [001] for $H=$ $0.05 \mathrm{~T}$. Rotating $\boldsymbol{H}$ in the (100) and (010) planes gives similar results. (d) Temperature dependence of the separated $\sin 2 \alpha$ and $\sin 4 \alpha$ contributions to the total torque shown in (c). (e) Field-evolution of the effective susceptibility $\chi(\alpha) \equiv \tau(\alpha) / H^{2}$ revealing the spin-flop transition at $T=2 \mathrm{~K}$.

\section{Theoretical modelling and phase diagram}

Combining the field and temperature dependence of $T_{N}$ from the heat capacity with the spin flops and $T_{a b}$ from the dc magnetization, we summarise our lowtemperature data in Fig. 5a. Little or no anisotropy is observed for different magnetic field orientations, as expected for a crystal exhibiting multiple orthogonal domains. The presence of $T_{a b}$ in this phase diagram indicates that single-ion anisotropy terms induced by the 


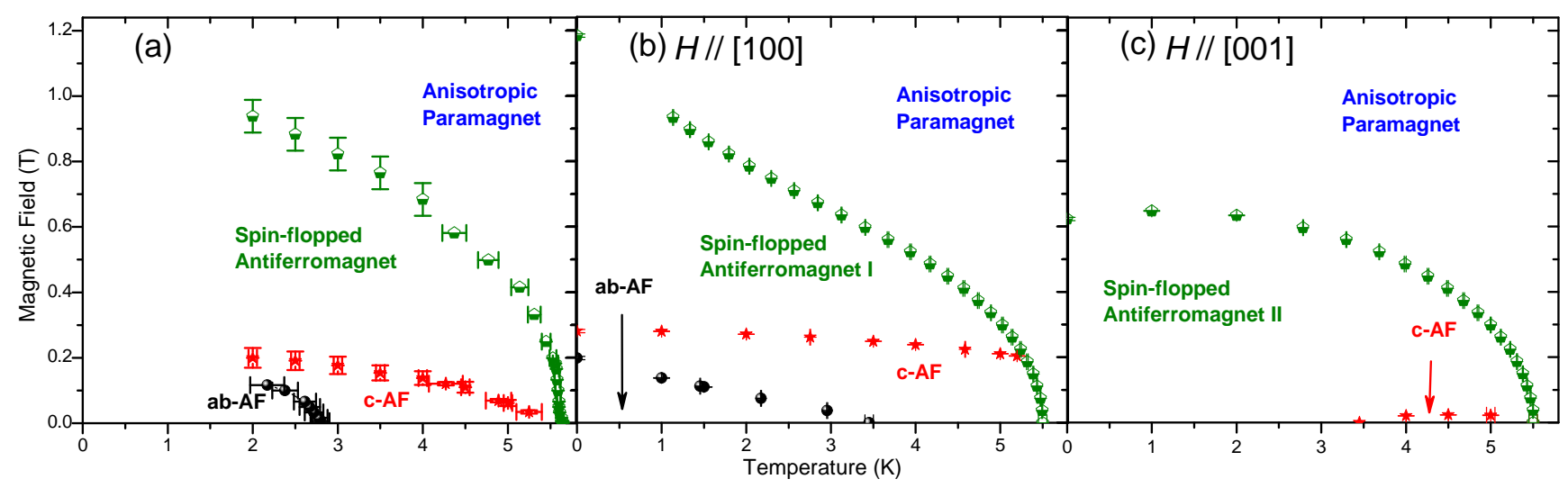

FIG. 5: (a) Experimentally-determined magnetic phase diagram of EuTiO3. (b,c) Two-sublattice mean-field phase diagrams for $\boldsymbol{H} / /$ [100] and [001] (see text for parameters). ab-AF refers to zero-field AF order along the [110] or [110] axes, while $c$-AF describes AF order along [001]. For the spin-flopped AF I phase, the spins order in the $a b$ plane with the staggered component of the magnetization perpendicular to the applied field. In contrast, spin-flopped AF II corresponds to AF ordering along [110] or $[1 \overline{1} 0]$, with a uniform magnetization component along the $c$-axis. The emergence of orthogonal domains at low temperature in $\mathrm{EuTiO}_{3}$ implies that our experimentally-determined diagram (a) should be a superposition of (b) and (c). Experimental evidence for the small pocket of $c$-AF in (c) is provided by the faint anomaly visible at low fields in $\chi(T)$ (indicated by an arrow in Fig. 3a.)

tetragonal crystal field in $\mathrm{EuTiO}_{3}$ play an important role at low temperature. To model the effect of these terms, we consider a classical Heisenberg model with nearest-neighbour $\left(J_{1}\right)$ and next-nearest-neighbour $\left(J_{2}\right)$ exchange interactions

$$
\begin{aligned}
\mathcal{H}= & J_{1} \sum_{\left\langle\boldsymbol{r}, \boldsymbol{r}^{\prime}\right\rangle} \boldsymbol{S}_{\boldsymbol{r}} \cdot \boldsymbol{S}_{\boldsymbol{r}^{\prime}}+J_{2} \sum_{\left\langle\left\langle\boldsymbol{r}, \boldsymbol{r}^{\prime}\right\rangle\right\rangle} \boldsymbol{S}_{\boldsymbol{r}} \cdot \boldsymbol{S}_{\boldsymbol{r}^{\prime}} \\
& +\sum_{\boldsymbol{r}} V\left(\boldsymbol{s}_{\boldsymbol{r}}\right)-\sum_{\boldsymbol{r}} \boldsymbol{B} \cdot \boldsymbol{S}_{\boldsymbol{r}}
\end{aligned}
$$

together with a single-ion anisotropy potential

$$
V(s)=-C_{2}\left(S_{x}^{2}+S_{y}^{2}\right)-C_{4}\left(S_{x}^{4}+S_{y}^{4}\right),
$$

where $S_{x}=\boldsymbol{S} \cdot(1,1,0) / \sqrt{2}$, and $S_{y}=\boldsymbol{S} \cdot(-1,1,0) / \sqrt{2}$. $\boldsymbol{S}_{\boldsymbol{r}}$ represents classical spin at $\boldsymbol{r}$ with $\left|\boldsymbol{S}_{\boldsymbol{r}}\right|=7 / 2$. The exchange parameters were determined by fitting the measured $T=0$ saturation field $H_{\text {sat }}=12 S J_{1} / g \mu_{B} \sim 1 \mathrm{~T}$ and Curie-Weiss temperature $T_{C W}=-2 S^{2}\left(J_{1}+2 J_{2}\right) \simeq$ $4 \mathrm{~K}^{5}$ ( $g \simeq 2$ is the gyromagnetic factor $)$, resulting in $J_{1} S^{2}=0.31 \mathrm{~K}$ and $J_{2} S^{2}=-1.22 \mathrm{~K}$. This yields a mean field $T_{N}=2 S^{2}\left(J_{1}-2 J_{2}\right) \sim 5.5 \mathrm{~K}$, in excellent agreement with the measured value. The single-ion anisotropy parameters $C_{2} S^{2}=-0.4 \mathrm{~K}$ and $C_{4} S^{4}=0.455 \mathrm{~K}$ were chosen to reproduce our experimental phase topology, especially the transition at $T_{a b} \sim 3 \mathrm{~K}$.

The phase diagrams obtained from a two-sublattice mean-field theory with magnetic field applied along the [100] and [001] directions are shown in Figs. 5b and c. Although our calculated $c$-AF region persists to slightly higher fields than our data, the overall agreement is reasonably good considering the limitations of a mean field approach.

\section{E. Electric field-mediated structural control}

The paraelectric nature of $\mathrm{EuTiO}_{3}$ provides a possible route to suppressing the orientational disorder below $T_{c}$. In $\mathrm{SrTiO}_{3}$, it is known that the application of an electric field $\boldsymbol{E}$ prevents softening for phonon wavevectors $\mathbf{k} / / \boldsymbol{E}^{27}$. Since a cubic-tetragonal phase transition entails phonon softening parallel to the tetragonal $c$ axis, cooling with an electric field $\boldsymbol{E}$ applied parallel to a cubic crystal axis should ensure that the eventual tetragonal $c$-axis lies perpendicular to $\boldsymbol{E}$, thus eliminating one of the three potential $c$-axis orientations. We may detect any change in the structure by measuring the variation in the low-temperature magnetization between $E$-fieldcooling (EFC) and zero-field-cooling in the same crystal: this principle is outlined in Fig. 6a and b. It is crucial to perform EFC from above $50 \mathrm{~K}$, since below this temperature the phonon softening terminates, quantum fluctuations dominate the low-energy phonon spectrum and the lattice structure is presumably locked.

Fig. 6c shows the effect of EFC on $\chi(H)$ for a $\mathrm{EuTiO}_{3}$ crystal cooled from $80 \mathrm{~K}$ : the spin-flop is clearly suppressed, implying that an increased proportion of our crystal now exhibits $c / / \boldsymbol{H}$. A maximum change in $\chi(H)$ below $0.05 \mathrm{~T}$ suggests that minor structural defects also contribute to the non-linear magnetization at low field. Domain walls provide one plausible origin for such defects; alternatively (and as suggested by XRD data ${ }^{13}$ ), it is possible that the structural disorder present in $\mathrm{EuTiO}_{3}$ at low temperature may be more complex than mere nanoscale twinning and the consequent orientational disorder revealed by our experiments.

Recent synchrotron experiments have indicated the presence of an incommensurate phase below $285 \mathrm{~K}$, due 

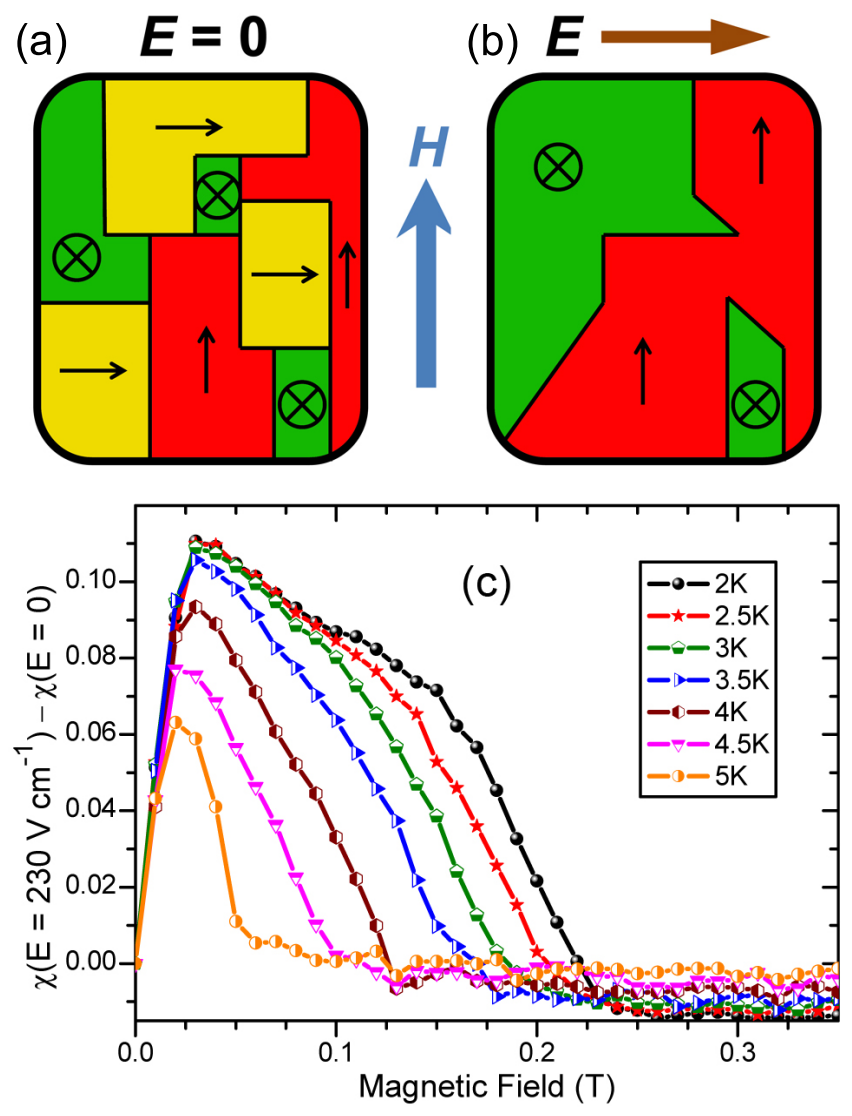

FIG. 6: Selective domain reorientation via electric field cooling in $\mathrm{EuTiO}_{3}$. (a) Zero-field-cooled $\mathrm{EuTiO}_{3}$ exhibits many orthogonal domains, whose $c$-axis orientations are indicated by arrows and shading. When a magnetic field $\boldsymbol{H}$ is applied, domains with $c \perp \boldsymbol{H}$ (shaded green and yellow) will undergo a spin-flop transition, generating a non-linearity in $M(H)$ at low field. (b) Cooling in an electric field E realigns structural domains with $c / / \boldsymbol{E}$, reducing the total proportion of the crystal with $c \perp \boldsymbol{H}$ and hence suppressing the spin-flop signal in $M(H)$. (c) Electric field-induced spin-flop suppression in an $\mathrm{EuTiO}_{3}$ crystal cooled from $T=80 \mathrm{~K}$ under $E=230 \mathrm{~V} \mathrm{~cm}^{-1}$ $(\boldsymbol{E} \perp \boldsymbol{H})$. To apply the field, gold contacts were sputtered on opposing crystal faces and wires attached with silver epoxy.

to competition between antiferrodistortive (AFD) and antiferroelectric (AFE) instabilities, with the tetragonal phase only emerging below $160 \mathrm{~K}^{28}$. The formation of this modulated structure was subsequently linked to the presence of $\mathrm{Eu}^{3+}$ ions and oxygen vacancies, both of which suppress $T_{N}{ }^{29}$. While competition between AFD and AFE instabilities may be partially responsible for the metastability seen in our specific heat data, we stress that our $T_{N}=5.6 \mathrm{~K}$ is the highest ever reported for $\mathrm{EuTiO}_{3}$. The incommensurate phase is therefore absent from our crystals and unrelated to the EFC effects discussed in our work.

In addition to confirming the presence of orthogonal domains (and their influence on the magnetic ground state), these results prove that we are able to control the domain orientation in $\mathrm{EuTiO}_{3}$ significantly below its

cubic-tetragonal phase transition. Future work should seek to identify whether a two-stage cooling process (using two orthogonal electric fields applied within distinct temperature ranges) is capable of totally suppressing disorder, i.e. achieving a single tetragonal domain.

The effects of varying the cooling rate through $T_{c}$ also remain to be explored, although it is uncertain whether this could influence the low temperature structure. To resolve this, it will be important to establish whether the structural transition is second order (as suggested by thermal expansivity data ${ }^{29}$ and analogous to $\mathrm{SrTiO}_{3}{ }^{10}$ ) or first order (with an associated change in the unit cell volume), since dynamic effects play a relatively minor role in the evolution of the order parameter below a second order phase transition and hence the low temperature structure should be independent of cooling rate for this case. A detailed dynamic analysis of the structural phase transition in $\mathrm{EuTiO}_{3}$ is beyond the scope of the present work. Nevertheless, it should be noted that several experiments with very different cooling rates have all yielded evidence for orientational domain misalignment: $d T / d t>10 \mathrm{~K} / \mathrm{min}^{-1}$ during a nitrogen streamcooled XRD experiment, compared with $\sim 1 \mathrm{~K} / \mathrm{min}^{-1}$ in a SQUID magnetometer and $<1 \mathrm{~K} / \mathrm{min}^{-1}$ for heat capacity measurements.

\section{CONCLUSIONS}

We have shown that monocrystalline $\mathrm{EuTiO}_{3}$ develops multiple orthogonal domains at low temperature, although its tetragonal ground state lacks any intrinsic structural, magnetic or electronic frustration. It is natural to question the effect of these domains on the dielectric properties of $\mathrm{EuTiO}_{3}$ : although neither the structural transition nor the AF order alone provide the spatial inversion symmetry-breaking necessary to stabilise ferroelectricity, it is plausible that disorder within the domain walls could cant the $\mathrm{Eu}^{2+}$ moments via the DM interaction. This would drive local symmetry-breaking and hence enable the formation of nanoscale electric dipoles. The approximately equal populations of the orthogonally-oriented domains and the random nature of the associated domain wall disorder together imply that the global dipole moment would average to zero, rendering FE order difficult to detect. Furthermore, any attempt to pole the crystal by applying an electric field will reduce the domain wall density and suppress the very disorder which generates the moments. Nevertheless, the possibility of achieving a static polarization extending over useful lengthscales via co-operative dipole freezing in $\mathrm{EFC}^{\mathrm{EuTiO}} 3$ merits further attention.

More generally, this mechanism of homogeneity control via electric field cooling may prove crucial for phase management in future devices made from elastically hard oxides. The possibility to continuously modulate the domain density and orientation in a paraelectric presents an attractive analogy to the piezoelectric effect in ferro- 
electrics, since the origin of these phenomena (the coupling of lattice dipoles to an external electric field) is identical. In future, we envisage continuously tuning material properties such as thermal conductivity via electric field-cooling. $\mathrm{EuTiO}_{3}$ was previously better known for its strain-modulated emergent multiferroicity: this fascinating material has now revealed another exciting method for phase control, with great potential for application in other paraelectrics.

\section{ACKNOWLEDGEMENTS}

We thank I. Martin and R. Lortz for useful discussions. This work was supported by The National Research Foundation of Singapore through the Competitive Research Programme (CRP Award No. NRF-CRP-42008-04), the European Union through MEXT-CT-2006039047 and EURYI research grants. Work at the LANL

was performed under the auspices of the U.S. DOE contract No. DE-AC52- 06NA25396 through the LDRD program. 
1 A. Ohtomo, D. Muller, J. Grazul, and H. Hwang, Nature 419, 378 (2002).

2 S.-Y. Chung, I.-D. Kim, and S.-J. Kang, Nat. Mater. 3, 774 (2004).

${ }^{3}$ Y. Kozuka, M. Kim, C. Bell, B. Kim, Y. Hikita, and H. Hwang, Nature 462, 487 (2009).

4 J. Lee, L. Fang, E. Vlahos, X. Ke, Y. Jung, L. Fitting Kourkoutis, J.-W. Kim, P. Ryan, T. Heeg, M. Roeckerath, et al., Nature 466, 954 (2010).

${ }^{5}$ T. McGuire, M. Shafer, R. Joenk, H. Alperin, and S. Pickart, J. Appl. Phys. 37, 981 (1966).

6 T. Katsufuji and H. Takagi, Phys. Rev. B 64, 054415 (2001).

7 C. Fennie and K. Rabe, Phys. Rev. Lett. 97, 267602 (2006).

8 R. Ranjan, H. Nabi, and R. Pentcheva, J. Phys.: Condens. Matter 19, 406217 (2007).

9 V. Shvartsman, P. Borisov, W. Kleeman, S. Kamba, and T. Katsufuji, Phys. Rev. B 81, 064426 (2010).

10 E. Salje, M. Gallardo, J. Jiménez, F. Romero, and J. del Cerro, J. Phys.: Condens. Matter 10, 5535 (1998).

11 A. Bussmann-Holder, J. Köhler, R. Kremer, and J. Law, Phys. Rev. B 83, 212102 (2011).

12 J. Köhler, R. Dinnebier, and A. Bussmann-Holder, Phase Transitions iFirst, 1 (2012).

13 M. Allieta, M. Scavini, L. Spalek, V. Scagnoli, H. Walker, C. Panagopoulos, S. Saxena, T. Katsufuji, and C. Mazzoli, Phys. Rev. B 85, 184107 (2012).

14 J. Bettis, M.-H. Whangbo, J. Köhler, A. BussmannHolder, and A. Bishop, Phys. Rev. B 84, 184114 (2011).

15 E. Dagotto, Science 309, 257 (2005).

16 T. Katsufuji and Y. Tokura, Phys. Rev. B 60, R15021 (1999).
17 A. Aharoni, J. Appl. Phys. 83, 3432 (1998).

18 A. Petrović, R. Lortz, G. Santi, M. Decroux, H. Monnard, Ø. Fischer, L. Boeri, O. Andersen, J. Kortus, D. Salloum, et al., Phys. Rev. B 82, 235128 (2010).

19 J. Lashley, M. Hundley, A. Migliori, J. Sarrao, P. Pagliuso, T. Darling, M. Jaime, J. Cooley, W. Hults, L. Morales, et al., Cryogenics 43, 369 (2003).

20 V. Goian, S. Kamba, J. Hlinka, P. Vanek, A. Belik, T. Kolodiazhnyi, and J. Petzelt, Eur. Phys. J. B 71, 429 (2009).

21 T. Wei, H. Liu, Y. Chen, H. Yan, and J.-M. Liu, Appl. Surf. Sci. 257, 4505 (2011).

${ }^{22}$ K. Kugimiya, K. Fujita, K. Tanaka, and K. Hirao, J. Magn. Magn. Mater. 310, 2268 (2007).

23 J. Chrosch and E. Salje, J. Phys.: Condens. Matter 10, 2817 (1998).

24 J. Lee, X. Ke, N. Podraza, L. Fitting Kourkoutis, T. Heeg, M. Roeckerath, J. Freeland, C. Fennie, J. Schubert, D. Muller, et al., Appl. Phys. Lett. 94, 212509 (2009).

25 G. Meijer, C. Rossel, E. Kopnin, M. Willemin, J. Karpinski, H. Schwer, K. Conder, and P. Wachter, Europhys. Lett. 42, 339 (1998).

${ }^{26}$ M. Herak, M. Miljak, G. Dhalenne, and A. Revcolevschi, J. Phys.: Condens. Matter 22, 026006 (2010).

27 J. Worlock and P. Fleury, Phys. Rev. Lett. 19, 1176 (1967).

28 J.-W. Kim, P. Thompson, S. Brown, P. Normile, J. Schlueter, S. A., A. Weidenkaff, and P. Ryan, arXiv:1206.5417 (2012).

29 V. Goian, S. Kamba, O. Pacherová, J. Drahokoupil, L. Palatinus, M. Dušek, J. Rohlíek, M. Savinov, F. Laufek, W. Schranz, et al., Phys. Rev. B 86, 054112 (2012). 